\title{
Rationale and design of the Edwards SAPIEN-3 periprosthetic leakage evaluation versus Medtronic CoreValve in transfemoral aortic valve implantation (ELECT) trial
}

\section{A randomised comparison of balloon-expandable versus self-expanding transcatheter aortic valve prostheses}

\author{
M. Abawi ${ }^{1}$ P. Agostoni ${ }^{1,2} \cdot$ N. H. M. Kooistra ${ }^{1} \cdot$ M. Samim² F. Nijhoff ${ }^{1}$ M. Voskuil $^{1} \cdot$ H. Nathoe $^{1}$ • \\ P. A. Doevendans ${ }^{1}$ - S. A. Chamuleau ${ }^{1}$ K. Urgel $^{1} \cdot$ J. Hendrikse $^{3} \cdot$ T. Leiner He A. C. Abrahams $^{4} \cdot$ B. van der $^{3}$ \\ Worp $^{5}$ - P. R. Stella ${ }^{1}$ \\ Published online: 9 December 2016 \\ (c) The Author(s) 2016. This article is available at SpringerLink with Open Access.
}

\begin{abstract}
Background and objectives Periprosthetic aortic regurgitation (PPR) after transcatheter aortic valve implantation (TAVI) remains an important issue associated with impaired long-term outcomes. The current randomised study aims to evaluate potential differences between the balloon-expandable Edwards SAPIEN-3 and the self-expanding Medtronic CoreValve system with the main focus on post-TAVI PPR by means of novel imaging endpoints, and an additional focus on other clinical endpoints.

Endpoints The primary endpoint of this study is quantitative assessment of the severity of post-procedural PPR using cardiac magnetic resonance imaging. Several other novel imaging modalities (X-ray contrast angiography, echocardiography) are used as secondary imaging modalities for the assessment of PPR following TAVI. Secondary objectives of the study include clinical outcomes such as cerebral and kidney injury related to TAVI, and quality of life.

Methods and design The ELECT study is a single-centre, prospective, two-armed randomised controlled trial. For the purpose of this study, 108 consecutive adult patients
\end{abstract}

P. R. Stella

p.stella@umcutrecht.nl

1 Department of Cardiology, University Medical Centre Utrecht, Utrecht, The Netherlands

2 Department of Cardiology, St. Antonius Hospital, Nieuwegein, The Netherlands

3 Department of Radiology, University Medical Centre Utrecht, Utrecht, The Netherlands

4 Department of Nephrology and Hypertension, University Medical Centre Utrecht, Utrecht, The Netherlands

5 Department of Neurology, University Medical Centre Utrecht, Utrecht, The Netherlands suitable for transfemoral TAVI will be randomly allocated to receive the SAPIEN-3 $(n=54)$ or the CoreValve system $(n=54)$.

Discussion The ELECT trial is the first randomised controlled trial to quantitatively compare the extent of postTAVI PPR between the SAPIEN-3 and CoreValve. Furthermore, it will evaluate potential differences between the two prostheses with regard to mid-term clinical outcome and quality of life.

Keywords Transcatheter aortic valve implantation • Periprosthetic aortic regurgitation - SAPIEN-3 - CoreValve

\section{Background}

Transcatheter aortic valve implantation (TAVI) is a valid treatment strategy for patients with severe symptomatic aortic stenosis who are regarded as being at high risk [1, 2] or as unable to undergo open-heart surgery [3]. Two transcatheter heart valves (THVs) based on different technical concepts have been developed and are widely used. These are the balloon-expandable SAPIEN THV (Edwards Lifesciences, Irvine, CA, USA), and the self-expanding CoreValve THV (CV-THV) (Medtronic, Minneapolis, MN, USA). Both THVs have shown excellent clinical results, but each has specific features, advantages and disadvantages [2, $4,5]$.

Currently available evidence suggests that TAVI is feasible and provides long-term haemodynamic and clinical improvements, but questions remain concerning the safety and durability of this technique. Several important complications of TAVI have to be addressed in order to warrant the wider use of this procedure [6, 7]. Significant concerns have been raised about the high incidence of post-procedu- 
ral periprosthetic aortic regurgitation (PPR) $[1,2]$ which is associated with increased mortality [8-11]. Other important procedural-related complications are cerebrovascular events [12], cardiac conduction disorders [13] and acute kidney injury [14, 15].

It has been estimated that between $41-100 \%$ of patients have some degree of PPR following TAVI (mild $>45 \%$; moderately severe 2-12\%) [10, 16]. Even mild PPR is associated with 10-15\% higher mortality after two years in comparison with patients with either no or only a trace of PPR, as shown in the PARTNER cohort A [4]. The extent and severity of native valve and left ventricular outflow tract (LVOT) calcification, undersizing of the valve prosthesis relative to the dimensions of the aortic annulus, suboptimal placement of the prosthesis and incomplete apposition of the stent frame owing to calcification in the device landing zone are all known mechanisms of PPR following TAVI [17]. However, the impact of prosthesis type (S3-THV or $\mathrm{CV}-\mathrm{THV}$ ) on the risk of post-TAVI PPR is less clear.

A few recent reports $[17,18]$, one of which was the only randomised trial to compare SAPIEN XT-THV (SXTTHV) (Edwards Lifesciences, Irvine, CA, USA) with CVTHV (CHOICE study), have suggested differences in the haemodynamic performance of both THVs where the use of CV-THV was associated with a higher rate of residual PPR. However, the operator's familiarity with the device and subjective measurement of PPR using unidimensional X-ray angiography images or two-dimensional (2D) echocardiography may bias the results. Hence, an adequately powered randomised study using quantitative assessment techniques may clarify the difference in severity of PPR between SXTTHV and CV-THV more reliably.

Although 2D echocardiography is currently considered the reference standard for evaluation and grading of aortic regurgitation, PPR is difficult to detect and quantify using conventional 2D echocardiography. In PPR, eccentric jets may become entrained along the left ventricular wall, which tends to alter their appearance and hence the perception of PPR severity [19]. Moreover, the possible presence of multiple jets originating from different periprosthetic locations makes their cumulative impact on the overall importance of PPR difficult to judge. Furthermore, in obese patients and patients with chronic obstructive pulmonary disease, the acoustic window at transthoracic echocardiography may be inadequate for comprehensive examination of the valves. Finally, this evaluation is also subject to inter- and intra-observer variability. For these reasons, other diagnostic modalities such as three-dimensional transoesophageal echocardiography (3D-TEE) [20, 21], phase contrast magnetic resonance imaging (MRI) [22], and X-ray contrast aortography [23] have been proposed for quantification of PPR after TAVI. These modalities can be used to measure the severity of aortic regurgitation by quantifying regurgi- tant volume and regurgitant fraction, thus minimising observer dependency.

Recently, the SAPIEN-3 THV (S3-THV) (Edwards Lifesciences, Irvine, CA, USA) was introduced [24] which, besides a differently designed stent frame, has an additional outer skirt in order to minimise post-TAVI PPR [5, 25]. However, whether this new feature will be effective in preventing post-TAVI PPR in daily practice remains to be established as well as the direct comparison versus the CV-THV. We designed a clinical trial for the randomised comparison of S3-THV and CV-THV in a routine TAVI population focusing primarily on post-TAVI PPR measured by different innovative imaging modalities, which allow a more accurate and observer-independent quantification of the PPR.

\section{Methods and design}

The ELECT study is a single-centre, prospective, twoarmed randomised controlled trial. Consecutive adult males or females, judged eligible for transfemoral TAVI by the local multidisciplinary heart team, and meeting the inclusion criteria will be approached for inclusion in this study. Device size selection will be based on charts provided by the manufacturer and on the pre-procedural multislice computed tomography (MSCT) annular perimeter. The ELECT trial has been designed in accordance with the ethical principles of the Declaration of Helsinki. The Ethics Committee of the University Medical Center Utrecht has given its full approval for the study and patients who agree to participate will be asked for written informed consent.

\section{Inclusion criteria}

In order to be eligible to participate in this study, subjects must meet all of the following criteria: The patient must be $\geq 18$ years of age and diagnosed with severe symptomatic aortic stenosis, judged inoperable or at high surgical risk (EuroSCORE $>15 \%$ or other criteria that make surgery high risk by a consensus among cardiologists and cardiac surgeons in the heart team) and deemed eligible for transfemoral TAVI. The diameter of the aortic annulus diameter should be $\geq 18$ and $\leq 28 \mathrm{~mm}$ assessed with MSCT. There should be no contraindications to study requirements such as MRI or TEE.

\section{Exclusion criteria}

Patients unable or unwilling to give informed consent are excluded from this study, as are patients whose aortic annulus diameter does not meet the inclusion criteria. 
Fig. 1 Flowchart of the study

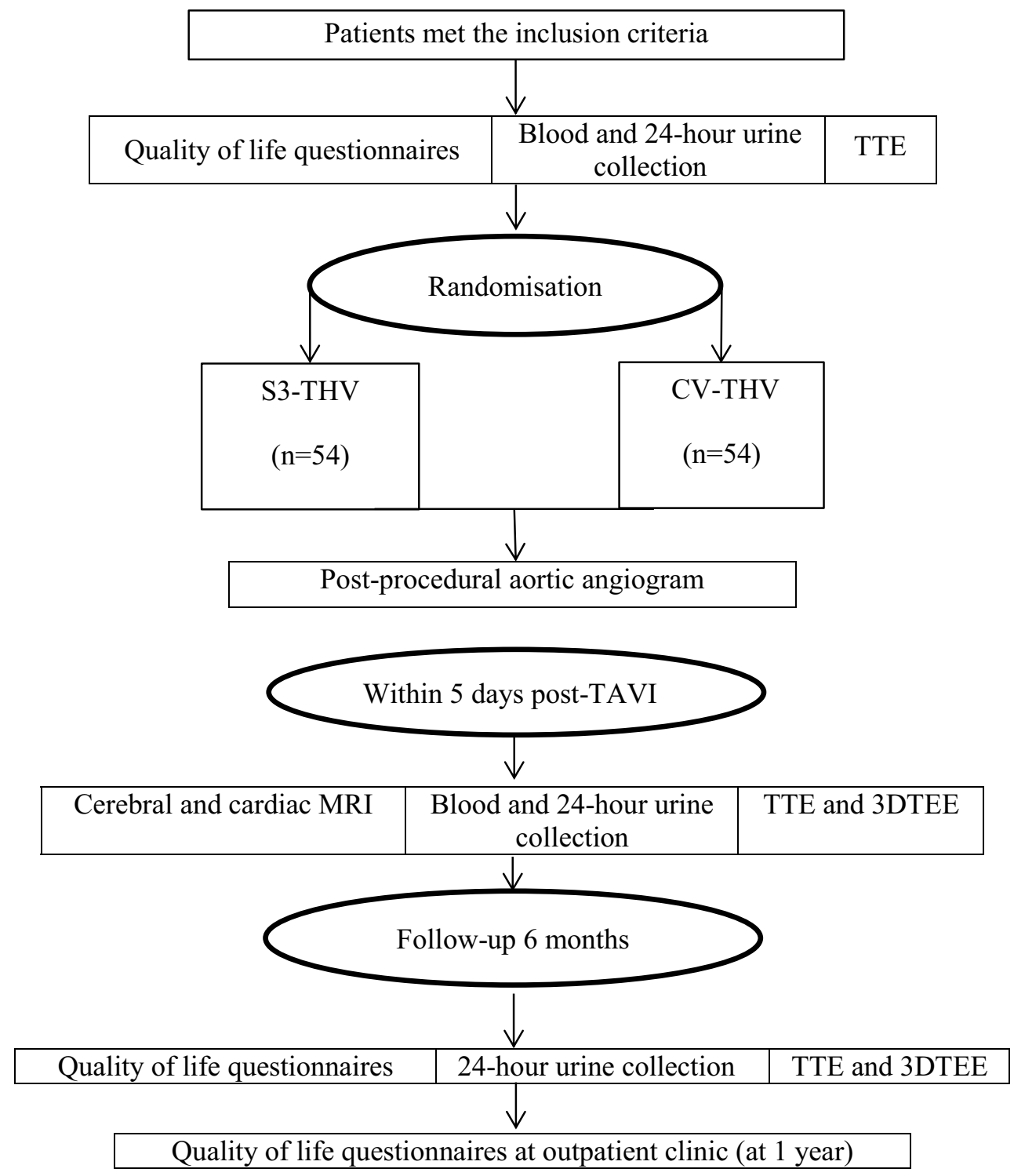

\section{Randomisation and interventions}

Patients who meet all the inclusion criteria and none of the exclusion criteria will be randomised in consecutive order. After successful puncture of the femoral artery chosen as entry site for TAVI, subjects will be randomised in a 1:1 fashion using sealed envelopes, to receive either an S3-THV or a CV-THV (Fig. 1).

Both prostheses (S3-THV and CV-THV) have received the CE mark of approval for the treatment of severe aortic stenosis. Determination of prosthesis size is based on the aortic annulus diameter, using pre-procedural contrastenhanced MSCT. The S3-THV is provided in three sizes: $23 \mathrm{~mm}, 26 \mathrm{~mm}$ and $29 \mathrm{~mm}$ (for native annulus diameters 18-22 mm, 21-25 mm, and 24-28 mm, respectively). The self-expanding CV-THV is available in four sizes: $23 \mathrm{~mm}$, $26 \mathrm{~mm}, 29 \mathrm{~mm}$ and $31 \mathrm{~mm}$ (for native aortic annulus di- ameters $18-20 \mathrm{~mm}, 20-23 \mathrm{~mm}, 23-27 \mathrm{~mm}$, and $27-28 \mathrm{~mm}$, respectively). In order to be able to randomise patients to receive S3-THV or CV-THV, only patients with an annular diameter range between $\geq 18$ and $\leq 28 \mathrm{~mm}$ will be included in this study.

\section{TAVI procedure}

All procedures will be performed by a highly experienced team with extensive knowledge of both devices. Details of the implantation technique for both S3-THVand CV-THV have been previously reported $[10,24,26,27]$. The procedure is routinely performed under conscious sedation with local anaesthesia. Fluoroscopy and intracardiac echocardiography are used for procedural guidance [28]. Balloon pre-dilatation is performed in all cases in accordance with our routine. Valve deployment is routinely done under rapid 


\section{Advertisement placed here.}

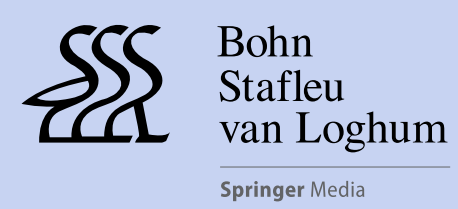

Houten 2017 


\section{Advertisement placed here.}

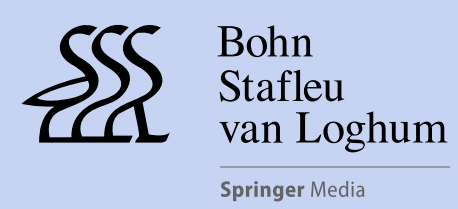

Houten 2017 


\section{Advertisement placed here.}

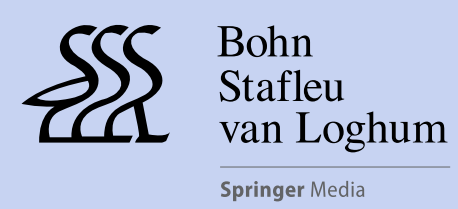

Houten 2017 


\section{Advertisement placed here.}

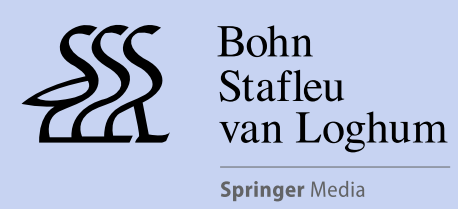

Houten 2017 
Fig. 2 X-ray contrast densitometry with the special software (CAAS A-Valve) after transcatheter aortic valve implantation

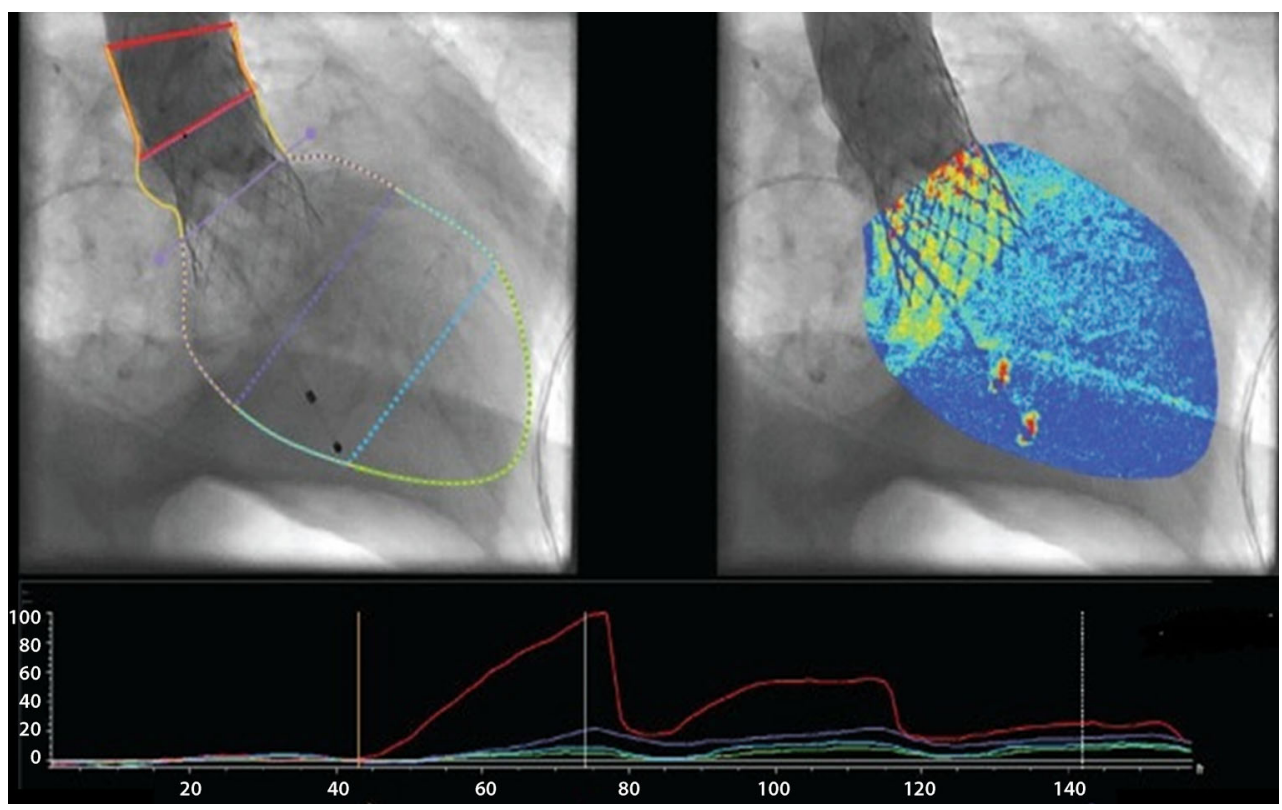

pacing (S3-THV 180 beats/min) or stabilising pacing (CVTHV 120 beats/min). Patients are monitored for at least $72 \mathrm{~h}$ and discharged on a regimen of lifelong low-dose aspirin (80-100 mg per day), or an oral anticoagulant (if there is a clinical indication for this), and 3 months of clopidogrel (75 mg per day).

\section{Endpoint assessment}

Follow-up assessments for the measurements of the primary and secondary endpoints will be performed at specified time points.

\section{Primary endpoint}

The primary endpoint of this study is quantitative assessment of the severity of post-procedural PPR using CMR. At day $4(+1)$ after TAVI, patients without contraindications for MRI will undergo cardiac phase contrast MRI for quantitative grading of PPR according to the standard grading criteria.

\section{Secondary endpoints}

The secondary endpoints of this study include assessment of PPR using other novel imaging modalities including $\mathrm{X}$-ray contrast angiography, and echocardiography (TTE and TEE). Furthermore, all clinical endpoints as defined by VARC-2 [29] and the quality of life questionnaires will be collected.

Contrast X-ray angiography is performed at the end of each TAVI procedure in order to measure the degree of postimplantation PPR by contrast densitometry (CAAS A-valve quantitative regurgitation analysis; Pie Medical Imaging, Maastricht, the Netherlands) (Fig. 2). Technical details of this approach have been reported previously [23].

Echocardiography, including TTE and TEE, is performed within five days following TAVI. The use of the Doppler technique provides accurate information on the number and severity of paravalvular jets. In addition, 3D TEE is used to analyse the complete morphology of the PPR colour flow stream in the region of its origin. Consequently, views from any level can be obtained, and the direction and extension of PPR jets can be assessed. New dedicated analysis software (Personal Space Technologies $\mathrm{BV}$, the Netherlands) designed for the visualisation and analysis of 3D volumetric echocardiography data will be used for the quantitative grading of PPR severity in this study.

Patients will undergo cerebral MRI including a diffusionweighted imaging sequence on day $4(+1)$ after TAVI, for detection of new cerebral ischaemic injury [30]. In order to detect kidney injury related to TAVI, serum and urine samples (including 24-hour urine) will be collected. Acute kidney injury will be assessed within 5 days after TAVI and compared with baseline. Irreversible kidney injury (VARC-2) will be assessed at 6-month follow-up. In order to investigate possible mid-term changes in post-TAVI PPR, valvular function will be evaluated at 6 months using TTE and 3D TEE as described above. Finally, validated quality of life questionnaires (KCC-Q, EuroQol EQ-5D and SF36) will be used to measure health-related quality of life at one year follow-up and compared with baseline (Fig. 3). 
Fig. 3 Planned flow diagram during ELECT trial

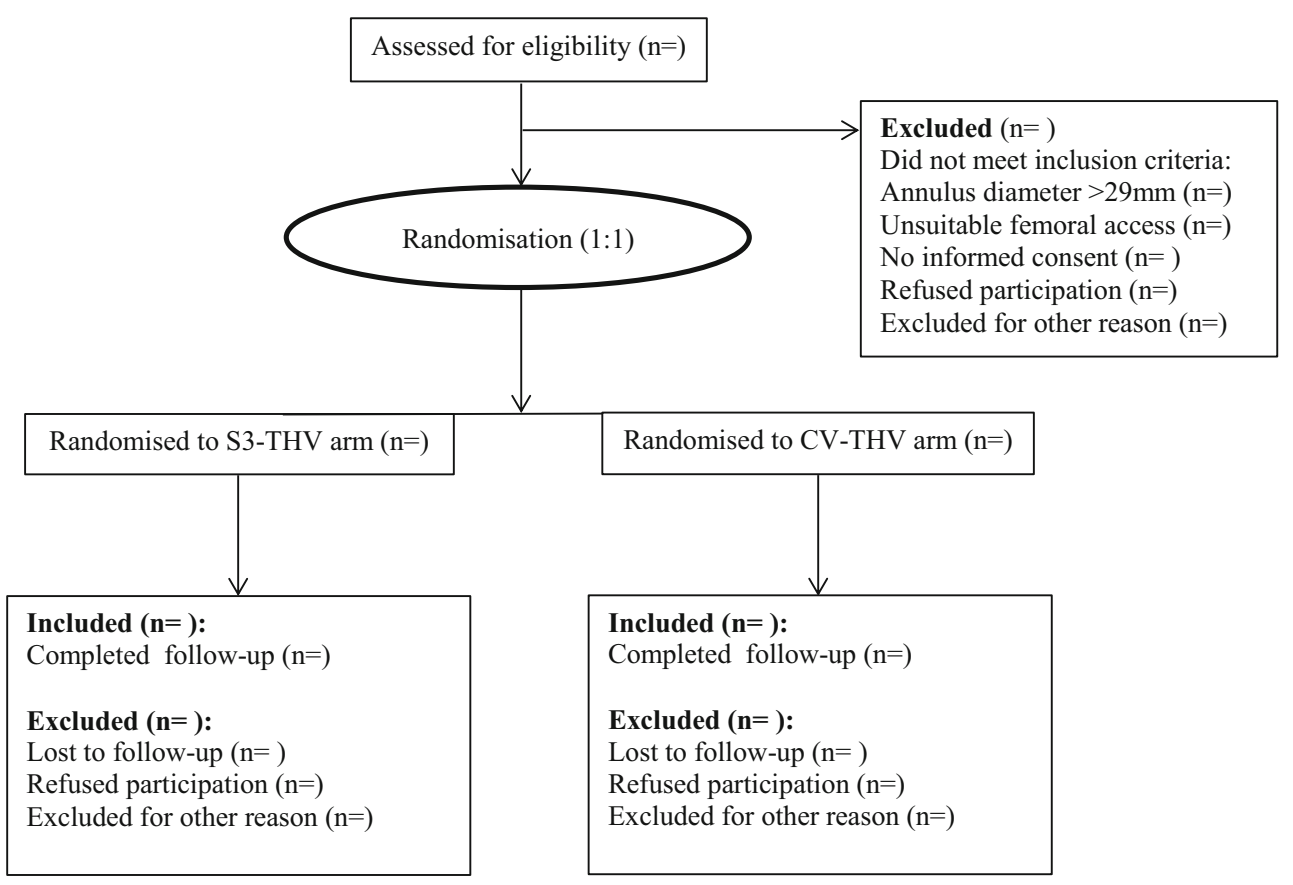

\section{Statistical analysis}

Descriptive statistics will be used to ascertain any imbalance between the arms at baseline. The extent of missing data will be reported and baseline factors will be compared for completers and non-completers to assess the extent of any bias that may result.

Continuous variables are presented as means \pm standard deviation or medians [interquartile range], as considered appropriate, and categorical variables as counts and percentages. Continuous variables will be compared using the Student $t$ test or Mann-Whitney test, depending on data distribution. Categorical variables will be compared using chisquare or Fisher's exact test, as considered appropriate. For the analysis of the difference in PPR between each of the prostheses, log transformation will be performed on the regurgitation volume data before performing a $t$ test, as these data are expected to be right-skewed. The follow-up analysis for changes in the endpoints in the time within the arms will be performed using the paired $t$ test and the repeated measurements analysis of variance. A two-tailed $p$-value of less than 0.05 is regarded as statistically significant. All analyses will be performed according to the intention-totreat principle. Data will be analysed using IBM SPSS Statistics software version 20 (IBM Corp., Armonk, NY, USA), and R version 2.12.0 (http://www.r-project.org).

\section{Sample size calculation}

A sample size calculation for the primary endpoint postTAVI PPR has been performed. A literature search on the difference in the extent of PPR after TAVI between the S3THV and CV-THV prostheses has been done. No studies were found that included a quantitative PPR comparison between these two prostheses. We used data from 25 patients treated with TAVI at our centre for a sample size calculation. PPR quantification in these patients was performed using cardiac MRI. Because the distribution of these volume data was skewed to the right, we performed a log transformation on the original data to improve approximations to normality (original volumes presented as $\mathrm{ml}$ ). The log transformed values were as follows: an overall mean regurgitant volume of 0.48 , an 'overall' median regurgitant volume of 0.48 , an overall standard deviation (SD) of 0.53 , and a mean regurgitant volume of 0.32 for S3-THV and a mean regurgitant volume of 0.63 for CV-THV. CMR grades of PPR will be defined according to regurgitation fraction using similar reference cut-point values according to the standard grading criteria. As even mild PPR has been shown to be associated with long-term mortality, any significant difference in postTAVI PPR volume between S3-THV and CV-THV is considered clinically important. The sample size calculation, including the above-mentioned log transformed values for mean regurgitant volumes and standard deviations, yielded a sample size of 49 patients in each arm to show superiority or equivalence over S3-THV and CV-THV with a power of $80 \%$. As we took an expected dropout rate of $10 \%$ into account, this yielded a total sample size of 54 patients in each arm. 


\section{Discussion}

The ELECT trial is the first randomised controlled trial to quantitatively compare the magnitude of post-procedural PPR between the S3-THV prosthesis and CV-THV. Other important objectives of this trial include assessment of the value of angiography, transthoracic and (3D) transoesophageal echocardiography and cardiac MRI for the measurement of post-TAVI PPR severity.

Regardless of the prosthesis type, post-procedural PPR is a common complication after TAVI associated with increased mortality [17]. A meta-analysis [25] including 45 studies reported an overall incidence of moderate or severe PPR of $11.7 \%$ within 30 days after TAVI. The balloonexpandable S3-THV [24] and self-expanding CV-THV [31] are two transcatheter heart valves that are in widespread use worldwide. Few previous studies comparing the haemodynamic performance of these two types of prosthesis have suggested a higher incidence of moderate or severe postTAVI PPR accompanying the implantation of CV-THV [1, 25].

An important aspect of CV-THV that might increase the risk of post-TAVI PPR (and thus explain the aforementioned data) is the intrinsic radial strength of its nitinol frame, which may not be sufficient for complete apposition of the prosthesis to the native annulus. This incomplete apposition might create periprosthetic gaps, especially in the presence of calcification along the aortic wall. Furthermore, an extreme angulation between the left ventricular outflow tract and the ascending aorta (also called the horizontal aorta) may reduce the ability of the CV-THV prosthesis to seal the paravalvular space [25]. Therefore, oversizing and balloon post-dilatation are more important in CV-THV implantation than in S3-THV implantation. In addition, owing to the non-cylindrical shape of the CV-THV system, its effective area inside the aortic annulus depends on the depth of the prosthesis in the left ventricular outflow tract. Therefore, in the CV-THV the sealing of the paravalvular space by the prosthesis also depends on the depth of its implantation. However, besides the factors mentioned above, which make CV-THV susceptible to post-TAVI PPR, we also need to keep in mind that the self-expanding nitinol frame has the potential to further expand over time. Therefore, the apposition of the prosthesis frame to the aortic annulus has the potential to improve gradually, decreasing the extent of post-TAVI PPR with passage of time.

Interestingly, the recently published results of the CoreValve US Pivotal Trial [32] showed that the incidence of any PPR after the implantation of CV-THV decreases over time: $41.1 \%$ at discharge and $31.9 \%$ at one year. The latter was also accompanied by a reduction in the incidence of more-than-mild aortic regurgitation during follow-up, at $13.8 \%$ at discharge, $10.1 \%$ at 6 months, and
$6.4 \%$ at one year. This suggests that measurement of postTAVI PPR in the CV-THV during the few first days after TAVI will probably overestimate its long-term severity. In the ELECT study, the severity of PPR is also measured at 6-month follow-up using transthoracic and 3D transoesophageal echocardiography, which allows for a more reliable comparison between the two prostheses.

One previously published meta-analysis [25] showed a higher risk of moderate or severe post-procedural PPR after CV-THV implantation (16\%) as compared with Edwards SAPIEN implantation $(9.1 \%, p=0.005)$. The CHOICE study [18], which is the only randomised headto-head trial to date that has compared the balloon-expandable SXT-THV with the CV-THV system, reported a significant difference in the frequency of any degree of PPR (38\% in SXT group versus 65\% in the CV group, $p<0.001)$ and more-than-mild PPR (4.1\% in SXT group versus $18.3 \%$ in the $\mathrm{CV}$ group, $p<0.001$ by angiography) favouring the balloon-expandable valve. However, the extent of the PPR in the CHOICE trial was measured using aortic root angiography immediately after prosthesis implantation, which is an important limitation of this clinical trial.

On the other hand, the PRAGMATIC [33] study reported a very low and comparable incidence of more-than-mild PPR with both Edwards SAPIEN and CV-THV (1.8\% and $2.0 \%$, respectively) in a large multicentre propensity scorematched study. Recently, another study among a selected group of patients receiving S3-THV or CV-THV reported a significantly lower rate of mild or severe PPR, lower need for permanent pacemaker implantation, and higher rate of device success for the S3-THV compared with CV-THV. However, this study was limited by its design and small sample size [34].

Obviously, there is a discrepancy in the incidence of PPR across different studies that is most probably related to the challenges in identification and quantification of post-TAVI PPR. Also, the VARC-2 document [29] did not propose new diagnostic criteria for adequate assessment of postTAVI PPR. The lack of a standardised and validated method for evaluation of post-TAVI PPR is a major limitation in comparing echocardiographic PPR analysis performed in different studies and meta-analyses. Aortic regurgitation after TAVI usually consists of multiple eccentric jets that are non-parallel and irregular in shape $[21,35]$.

Eccentric jets are frequently entrained along the left ventricular wall with fanning of jets as they regurgitate. Therefore, the eccentric aspects of post-TAVI PPR make the assessment of its severity challenging. Also acoustic shadowing from the calcifications and Doppler attenuation from the prosthesis can obscure regurgitant jets and thus result in underestimation of their severity [35]. 
On the other hand, assessment of PPR severity using aortic root angiography relies on subjective assessment of unidimensional images, and can be affected by inter-observer and intra-observer variability.

In the ELECT trial, the severity of PPR is measured quantitatively using several different modalities, from dedicated software for angiography [23] directly post-TAVI implantation to either transthoracic and transoesophageal echocardiography (with $3 \mathrm{D}$ reconstruction) $[20,21]$ to cardiac MRI including phase contrast sequences [22]. The main focus of the ELECT trial will be the comparison of the different imaging modalities in assessing PPR, using CMR as a primary endpoint. This comparison is an important aspect of the present trial as to date there is no validated tool for reliable measurement of post-TAVI PPR.

Funding Local cardiology R\&D department of the University Medical Centre Utrecht, the Netherlands (major) with minor additional research grants from Edwards (Lifesciences, Irvine, CA, USA) and Medtronic (Medtronic, Minneapolis, MN, USA).

Conflict of interest P. R. Stella is a physician-proctor for Edwards Lifesciences and Medtronic and is a member of the scientific advisory board of Keystone Heart. M. Abawi, P. Agostoni, N.H.M. Kooistra, M. Samim, F. Nijhoff, M. Voskuil, H. Nathoe, P. A. Doevendans, S. A. Chamuleau, K. Urgel, J. Hendrikse, T. Leiner, A. C. Abrahams and $\mathrm{B}$. van der Worp declare that they have no competing interests.

Open Access This article is distributed under the terms of the Creative Commons Attribution 4.0 International License (http:// creativecommons.org/licenses/by/4.0/), which permits unrestricted use, distribution, and reproduction in any medium, provided you give appropriate credit to the original author(s) and the source, provide a link to the Creative Commons license, and indicate if changes were made.

\section{References}

1. Smith CR, Leon MB, Mack MJ, et al. Transcatheter versus surgical aortic-valve replacement in high-risk patients. N Engl J Med. 2011;364:2187-98.

2. Adams DH, Popma JJ, Reardon MJ, et al. Transcatheter aorticvalve replacement with a self-expanding prosthesis. N Engl J Med. 2014;370(10):1790-8.

3. Leon MB, Smith CR, Mack M, et al. Transcatheter aortic-valve implantation for aortic stenosis in patients who cannot undergo surgery. N Engl J Med. 2010;363:1597-607.

4. Kodali SK, Williams MR, Smith CR, et al. Two-year outcomes after transcatheter or surgical aortic-valve replacement. N Engl J Med. 2012;366:1686-95.

5. Webb J, Gerosa G, Lefevre T, et al. Multicenter evaluation of a next-generation balloon-expandable transcatheter aortic valve. $\mathrm{J}$ Am Coll Cardiol. 2014;64:2235-43.

6. Rofe MT, Shacham Y, Steinvi A, et al. Prevalence of blood type A and risk of vascular complications following transcatheter aortic valve implantation. Neth Heart J. 2016;24(5):350-4.

7. Genereux P, Head S, Van Mieghem N, et al. Clinical outcomes after transcatheter aortic valve replacement (TAVR) using valve academic research consortium (VARC) definitions: A weighted metaanalysis of 3,519 patients from 16 studies. J Am Coll Cardiol. 2012;59:2317-26.
8. Gotzmann M, Korten M, Bojara W, et al. Long-term outcome of patients with moderate and severe prosthetic aortic valve regurgitation after transcatheter aortic valve implantation. Am J Cardiol. 2012;110:1500-6.

9. Kodali S, Pibarot P, Douglas PS, et al. Paravalvular regurgitation after transcatheter aortic valve replacement with the Edwards Sapien Valve in the PARTNER trial: characterizing patients and impact on outcomes. Eur Heart J. 2015;36:449-56.

10. Sinning J-M, Hammerstingl C, Vasa-Nicotera M, et al. Aortic Regurgitation Index defines severity of peri-prosthetic regurgitation and predicts outcome in patients after transcatheter aortic valve implantation. J Am Coll Cardiol. 2012;59:1134-41.

11. Moat NE, Ludman P, de Belder MA, et al. Long-term outcomes after transcatheter aortic valve implantation in high-risk patients with severe aortic stenosis. J Am Coll Cardiol. 2011;58:2130-8.

12. Nombela-Franco L, Webb JG, de Jaegere PP, et al. Timing, predictive factors, and prognostic value of cerebrovascular events in a large cohort of patients undergoing transcatheter aortic valve implantation. Circulation. 2012;126:3041-53.

13. Siontis GC, Juni P, Pilgrim T, et al. Predictors of permanent pacemaker implantation in patients with severe aortic stenosis undergoing TAVR: a meta-analysis. J Am Coll Cardiol. 2014;64:129-40.

14. Chatani K, Abdel-Wahab M, Wubken-Kleinfeld N, et al. Acute kidney injury after transcatheter aortic valve implantation: Impact of contrast agents, predictive factors, and prognostic importance in 203 patients with long-term follow-up. J Cardiol. 2015;66:514-9.

15. Pyxaras SA, Zhang Y, Wolf A, Schmitz T, Naber CK. Effect of varying definitions of contrast-induced acute kidney injury and left ventricular ejection fraction on one-year mortality in patients having transcatheter aortic valve implantation. Am J Cardiol. 2015;116:426-30.

16. Abdel-Wahab M, Zahn R, Horack M, et al. Aortic regurgitation after transcatheter aortic valve implantation: incidence and early outcome. Results from the German transcatheter aortic valve interventions registry. Heart. 2011;97:899-906.

17. Athappan G, Patvardhan E, Tuzcu EM, et al. Incidence, predictors, and outcomes of aortic regurgitation after transcatheter aortic valve replacement: meta-analysis and systematic review of literature. J Am Coll Cardiol. 2013;61:1585-95.

18. Abdel-Wahab M, Mehilli J, Frerker C, et al. Comparison of balloon-expandable vs self-expandable valves in patients undergoing transcatheter aortic valve replacement: the CHOICE randomized clinical trial. JAMA. 2014;311(15):1503-14.

19. Cape EG, Yoganathan AP, Weyman AE, Levine RA. Adjacent solid boundaries alter the size of regurgitant jets on doppler color flow maps. J Am Coll Cardiol. 1991;17(5):1094-102.

20. Smith LA, Dworakowski R, Bhan A, et al. Real-time threedimensional transesophageal echocardiography adds value to transcatheter aortic valve implantation. J Am Soc Echocardiogr. 2013;26:359-69.

21. Zamorano JL, Badano LP, Bruce C, et al. EAE/ASE recommendations for the use of echocardiography in new transcatheter interventions for valvular heart disease. Eur Heart J. 2011;32:2189-214.

22. Sherif MA, Abdel-Wahab M, Beurich HW, et al. Haemodynamic evaluation of aortic regurgitation after transcatheter aortic valve implantation using cardiovascular magnetic resonance. EuroIntervention. 2001;7:57-63.

23. Schultz CJ, Slots TL, Yong G, et al. An objective and reproducible method for quantification of aortic regurgitation after TAVI. EuroIntervention. 2014;10:355-63.

24. Binder RK, Rodés-Cabau J, Wood DA, Webb JG. Edwards SAPIEN 3 valve. EuroIntervention. 2012;8(Suppl Q):83-7.

25. Nijhoff F, Abawi M, Agostoni P, et al. Transcatheter aortic valve implantation with the new balloon-expandable Sapien 3 versus Sapien XT Valve System: A propensity score - matched singlecenter comparison. Circ Cardiovasc Interv. 2015;8:1-7. 
26. Webb JG, Chandavimol M, Thompson CR, et al. Percutaneous aortic valve implantation retrograde from the femoral artery. Circulation. 2006;113:842-50.

27. Nijhoff F, Agostoni P, Samim M, et al. Optimisation of transcatheter aortic balloon-expandable valve deployment: the twostep inflation technique. EuroIntervention. 2013;9:555-63.

28. Samim M, Agostoni P, Nijhoff F, et al. Three-dimensional aortic root reconstruction derived from rotational angiography for transcatheter balloon-expandable aortic valve implantation guidance. Int J Cardiol. 2014;176:1318-20.

29. Kappetein AP, Head SJ, Genereux P, et al. Updated standardized endpoint definitions for transcatheter aortic valve implantation: the Valve Academic Research Consortium-2 consensus document (VARC-2). Eur J Cardiothorac Surg. 2012;42:S45-S60.

30. Samim M, Hendrikse J, van der Worp HB, et al. Silent ischemic brain lesions after transcatheter aortic valve replacement: lesion distribution and predictors. Clin Res Cardiol. 2015;104:430-8.

31. Grube E, Laborde JC, Gerckens U, et al. Percutaneous implantation of the CoreValve self-expanding valve prosthesis in high-risk patients with aortic valve disease: the Siegburg first-in-man study. Circulation. 2006;114:1616-24.

32. Reardon MJ, Adams DH, Coselli JS, et al. Self-expanding transcatheter aortic valve replacement using alternative access sites in symptomatic patients with severe aortic stenosis deemed extreme risk of surgery. J Thorac Cardiovasc Surg. 2014;148:2869-76.

33. Chieffo A, Buchanan GL, Van Mieghem NM, et al. Transcatheter aortic valve implantation with the Edwards SAPIEN versus the Medtronic Corevalve Revalving system devices. J Am Coll Cardiol. 2013;61:830-6.

34. Gonska B, Seeger J, Baarts J, et al. The balloon-expandable Edwards Sapien 3 valve is superior to the self-expanding Medtronic CoreValve in patients with severe aortic stenosis undergoing transfemoral aortic valve implantation. J Cardiol. 2016; doi:10.1016/j. jjcc.2016.08.008.

35. Raffa GM, Malvindi PG, Settepani F, et al. Aortic valve replacement for paraprosthetic leak after transcatheter implantation. J Cardiac Surg. 2012;27:47-51. 